\title{
Capillary rise in nesting cylinders
}

\author{
Victor Brady*, Paul Concus ${ }^{\dagger}$, and Robert Finn ${ }^{\ddagger}$
}

\begin{abstract}
We investigate computationally recent results concerning the question of whether liquid necessarily rises higher in a capillary tube of smaller section, when tubes are placed vertically in an infinite reservoir. The numerical results corroborate for a particular example a striking discontinuous behavior that was predicted mathematically.
\end{abstract}

\section{Introduction}

For capillary tubes of circular section, it is generally known that a narrower tube when dipped vertically into an infinite reservoir raises a wetting liquid higher than does a wider tube, the rise height at the axis being essentially inversely proportional to tube radius [5], [3, Sec. 2.2]; see Fig. 1. In fact, it turns out that the surface height in the narrower tube is greater than the surface height in the wider tube at each point of the narrower tube's section. This is so even if the sections are not concentric [6] [3, Theorem 5.10]; see Fig. 2.

About thirty years ago M. Miranda posed informally the question of whether such a result might hold for tubes of general section: Does liquid

\footnotetext{
* Lawrence Berkeley National Laboratory, Berkeley, CA 94720

${ }^{\dagger}$ Lawrence Berkeley National Laboratory and Department of Mathematics, University of California at Berkeley, Berkeley, CA 94720

${ }^{\ddagger}$ Department of Mathematics, Stanford University, Stanford, CA 94305
} 


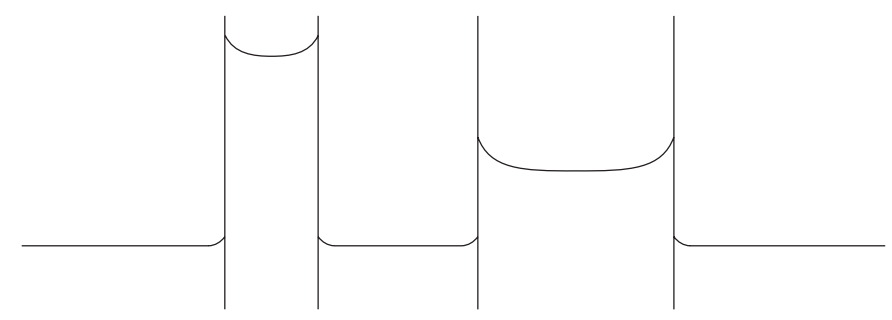

Figure 1: Narrower circular capillary tube raises liquid higher at its axis than does the wider tube at its axis.
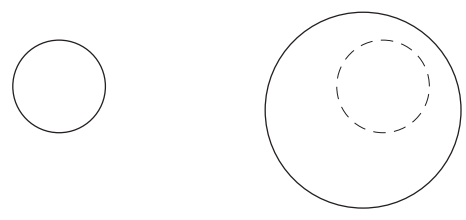

Figure 2: At each point of the narrower circular tube liquid rises higher than it does in the wider circular tube over the same section (dashed subdomain), even if the sections are not concentric.

in $\Omega_{\alpha}$ always rise higher than does liquid raised by $\Omega_{\beta} \supset \Omega_{\alpha}$ over the same section $\Omega_{\alpha}$ (Fig. 3)? A number of criteria for a positive answer were established in [6] and in [3, Sec. 5.3]. In contrast, [2] and [3, Sec. 5.4] distinguished special cases in which negative answers occur. Thus, the tube with larger section may indeed lift liquid higher over $\Omega_{\alpha}$ for some configurations.

More recently it was shown in [4] that even for sections of simple form the larger tube can lift liquid higher in low-gravity configurations. Furthermore, the height differences can become arbitrarily large as the gravity decreases to zero. Most strikingly, a discontinuous change in behavior can occur for infinitesimal changes in section. In the present paper we investigate this 

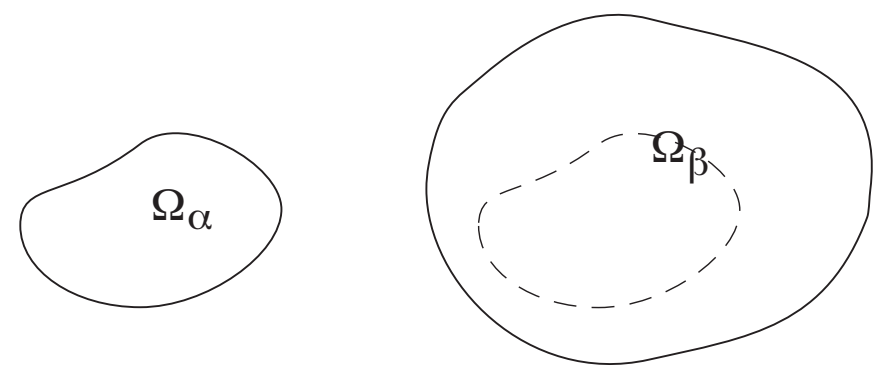

Figure 3: General tubes with nesting sections. Liquid need not rise higher in $\Omega_{\alpha}$ than in $\Omega_{\beta}$ over the same section (dashed subdomain).

behavior computationally for a particular family of sections given in [4].

\section{Governing Equations}

The governing equations for an equilibrium liquid-vapor interface in a vertical tube of section $\Omega$ in a downward-acting gravity field $(B>0)$ are

$$
\begin{gathered}
\operatorname{div} T u=B u \text { in } \Omega, \quad T u=\frac{\nabla u}{\sqrt{1+|\nabla u|^{2}}}, \\
\nu \cdot T u=\cos \gamma \text { on } \Sigma .
\end{gathered}
$$

The equations have been written in dimensionless form with $u$ being the ratio of the rise height above the surface level at infinity, to a characteristic length $a ; B=\rho g a^{2} / \sigma$ is the Bond number, with $\rho$ the density difference across the liquid-vapor interface, $\sigma$ the interfacial surface tension, and $g$ the acceleration due to gravity; $\Sigma$ is the boundary of $\Omega$, and $\nu$ is the outward unit normal on $\Sigma$; $\gamma$ denotes the contact angle between the liquid and the wall of the tube, with $0 \leq \gamma<\frac{\pi}{2}$ (wetting liquid). 


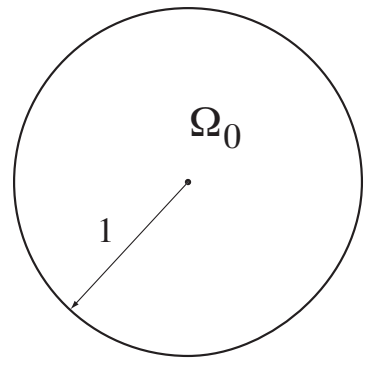

(a)

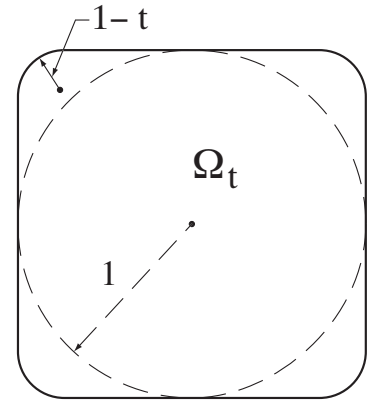

(b)

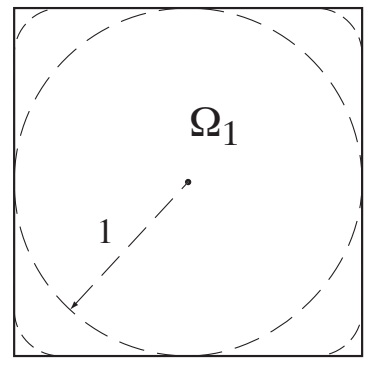

(c)

Figure 4: Sections for example problem. (a) unit disk $\Omega_{0}$, (b) circumscribing rounded squares $\Omega_{t}(0<t<1)$ showing $\Omega_{0}$ as dashed subdomain, and (c) $2 \times 2$ square $\Omega_{1}$ showing $\Omega_{0}$ and $\Omega_{t}$ as dashed subdomains.

The sections $\Omega$, presented in [4], for which we compute solutions of (1),(2) are depicted in Fig. 4. In dimensionless form $\Omega_{0}$ is the unit disk, and $\Omega_{1}$ is its circumscribing $2 \times 2$ square. These are the bounding members of a family of domains $\Omega_{t}$ obtained by smoothing the corners of $\Omega_{1}$ with circular arcs of radius $(1-t), 0 \leq t \leq 1$. The domains $\Omega_{t}$ with $0<t<1$, intermediate between the disk and the circumscribing square, are given the name disq domains in [4]; we adopt here the same terminology. Questions of existence and boundedness as $B \rightarrow 0$ are discussed in [4].

The following properties (i)-(iv) of solutions of (1),(2) are proved in [4] to hold whenever $\gamma \geq \pi / 4$. These form the basis for our computational study.

(i) For each of the disq domains $\Omega_{t}$, if $B$ is small enough, a capillary tube with square section $\Omega_{1}$ will raise liquid to a higher level over all 
of $\Omega_{t}$ than will $\Omega_{t}$ itself. Furthermore, for each fixed $t$ the difference in heights will tend to infinity like $1 / B$ as $B \rightarrow 0$.

Thus, in a comparison for small enough $B$ between the square and any disq, one has that the larger domain (the square) lifts liquid higher than the smaller one, arbitrarily higher as $B \rightarrow 0$.

In contrast there holds:

(ii) For any $B>0$ the height in the inscribed disk $\Omega_{0}$ exceeds at each point of $\Omega_{0}$ the height for the square and thus, ipso facto, the height for any of the intermediate $\Omega_{t}$ domains.

Thus, in a comparison between the disk $\Omega_{0}$ and any of the other domains, disq or square, one has that the smaller domain (the disk) lifts liquid higher than the larger one, arbitrarily higher in the case of a disq. This is the situation no matter how close the disq is to being the disk $\Omega_{0}$.

One thus finds that, having chosen any $B$ small enough so that (i) holds for a given disq domain $\Omega_{t}$ (square lifts liquid higher than disq does), the height inequality will nevertheless reverse if, for that fixed $B$, the disq $\Omega_{t}$ approaches the disk $\Omega_{0}$. That happens no matter how small $B$ is initially chosen.

In [4] these contrasting results are considered from the point of view of the two limiting behaviors: (a) as $t \rightarrow 0$ (domains changing from square to disk) for fixed $B$, and (b) as $B \rightarrow 0$ for fixed domain ( $t$ fixed). When both limits are taken the height inequality reversal transforms into a discontinu- 
ous dependence on the parameter $t$. Specifically, let $u_{t}$ denote the solution of (1),(2) in $\Omega_{t}$. One obtains for the limits of the height difference between the square and the other domains that

$$
\lim _{B \rightarrow 0} \lim _{t \rightarrow 0}\left(u_{1}-u_{t}\right)=C, \quad C<0,
$$

but that

$$
\lim _{t \rightarrow 0} \lim _{B \rightarrow 0}\left(u_{1}-u_{t}\right)=+\infty .
$$

(The negative constant $C$ is the height difference between the $B=0$ (spherical) solutions for the square and for the disk, discussed in Secs. 3 and 4.)

Thus, despite the apparently smooth domain transition from $\Omega_{t}$ to $\Omega_{0}$, the limiting procedures cannot be interchanged. Summarizing the substance of this seemingly anomalous behavior, we have from [4]:

(iii) Although the surface height at a given point is continuous in $t$ at $t=0$ for each fixed $B>0$, its limiting behavior as $B \rightarrow 0$ changes discontinuously in $t$ at $B=0$.

We shall corroborate these properties by the computations presented in Sec. 4. The computations illustrate also another result of [4]:

(iv) Any modification of one of the disq domains that decreases its area to that of the disk (and preserves the existence of solutions of $(1),(2))$ will reverse the conclusion of (ii) that the disk $\Omega_{0}$ lifts liquid higher than the disqs $\Omega_{t}$. The shrunken disq domain will not only lift 
liquid higher for small enough $B$, but the height differences become unboundedly large for decreasing $B$.

\section{Computational Procedure}

We are interested in solving (1),(2) for values of $B$ that may be very small and for which surface heights, which can grow like $1 / B$, may be very large. To control the magnitude of quantities being computed and the corresponding possible loss of significance from subtracting nearly equal large numbers, we make a shift of origin as follows.

Integrating (1) by parts over $\Omega$ and using (2), one obtains that $\bar{u}$, the average height of $u$, is

$$
\bar{u}=\frac{|\Sigma| \cos \gamma}{B|\Omega|}
$$

Here $|\Omega|$ denotes the area of $\Omega$, and $|\Sigma|$ denotes its perimeter. We shift $u$ by its average height to define the new variable $w$,

$$
w=u-\frac{|\Sigma| \cos \gamma}{B|\Omega|}
$$

Then, as equations for $w,(1),(2)$ become

$$
\begin{gathered}
\operatorname{div} T w=B w+\frac{|\Sigma| \cos \gamma}{|\Omega|} \text { in } \Omega, \quad T w=\frac{\nabla w}{\sqrt{1+|\nabla w|^{2}}} \\
\nu \cdot T w=\cos \gamma \text { on } \Sigma .
\end{gathered}
$$

One calculates that the average value $\bar{w}$ of $w$ is

$$
\bar{w}=0 .
$$


Hence, in connection with the result of D. Siegel [7] for smooth domains, that $u$ has asymptotic behavior as $B \rightarrow 0$

$$
u=\frac{|\Sigma| \cos \gamma}{B|\Omega|}+w+O(B)
$$

one can expect that using $w$ as independent variable will be favorable for computation for small $B$. This view is consistent with upper and lower bounds on $w$ that are given in [4].

We shall have use below for solutions of $(5),(6)$ for the limiting case of $B=0$ for the disk $\Omega_{0}$ and the square domain $\Omega_{1}$. For both domains, $|\Sigma| /|\Omega|=2$. The solutions are portions of the lower hemisphere

$$
w=\text { const. }-\sqrt{\sec ^{2} \gamma-r^{2}}
$$

where $r$ is the radial coordinate measured from the center of the domains. For $\gamma=60^{\circ}$, the contact angle used in our numerical examples, the values of the constant corresponding to $\bar{w}=0$ are $\frac{2}{3}(8-3 \sqrt{3}) \approx 1.869$ for $\Omega_{0}$ and approximately 1.822 for $\Omega_{1}$. A closed-form expression for the difference of the constants for more general $\gamma$ is given in [4].

For all the domains in our study we will need the values of $|\Sigma| /|\Omega|$. A straightforward calculation gives

$$
|\Sigma| /|\Omega|=2 \frac{\pi+t(4-\pi)}{\pi+t(2-t)(4-\pi)}
$$

This quantity is depicted in Fig. 5 as a function of $t$. Note that it achieves its largest value 2 for the disk $(t=0)$ and the square $(t=1)$ but is less for 


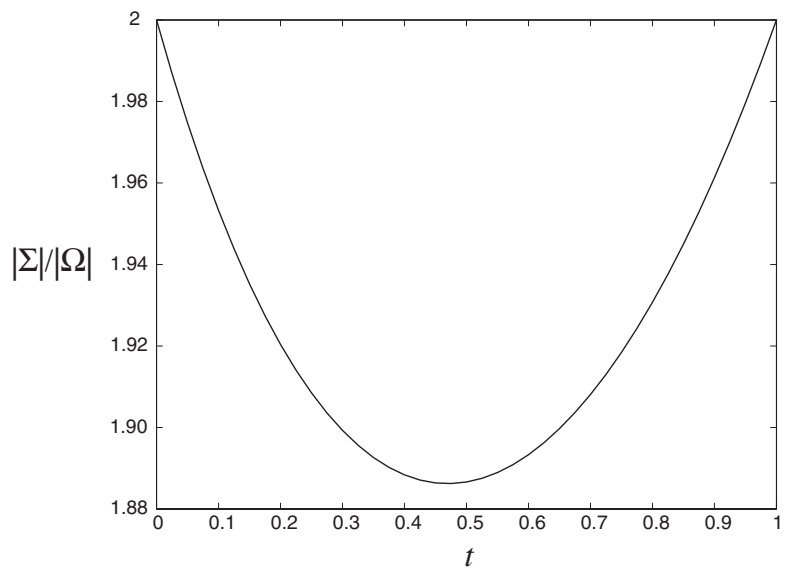

Figure 5: $|\Sigma| /|\Omega|$ vs. $t$.

the disqs $(0<t<1)$. Even though differences in $|\Sigma| /|\Omega|$ may be small for different domains, the differences in mean height (3) can become large as $B \rightarrow 0$. The results described in Sec. 2 can be seen to correlate with this behavior of the mean heights. The behavior is central to the mathematical approach in [4].

We used the software package PLTMG for obtaining numerical solutions of (5),(6). PLTMG is a general-purpose package for finite-element solution of certain second-order linear or nonlinear elliptic-partial-differential-equation boundary-value problems. It has numerous features including automatic refinement of the approximating triangular mesh, automatic error estimation, and, of particular use for our problem, spectral information on the underlying Jacobian matrices [1]. 


\section{Results for Example Problem}

Equilibrium free-surface configurations were calculated for domains $\Omega_{t}$ with $t=0,0.1,0.2, \ldots, 1.0$, for four values of Bond number $B=100,1,0.01$, 0.0001, and for contact angle $\gamma=60^{\circ}$. In general, the a posteriori error estimates for $w$ on the mesh used for most domains (approximately 4,000 vertices) indicated an accuracy in the solution of at least two or three decimal places.

\subsection{Ill Conditioning}

For the smallest Bond number $B=0.0001$, and to some extent for $B=$ 0.01, special considerations were required because of the problem's nearsingularity. For $B=0$ a solution is determined only up to an additive constant. Correspondingly the Jacobian matrix (positive-definite symmetric) of the discrete problem is near-singular for $B=0.0001$ with the near-zero smallest eigenvalue corresponding to the constant eigenvector (a multiple of the vector of all ones). This feature manifested itself most significantly for the curved domains, for which discretization errors associated with the curved boundaries resulted in a substantial component of the nearby null space being introduced into the solution. The following discussion indicates this phenomenon.

The numerical solution was obtained for Bond number $B=0.0001$ for the disk $(t=0)$ and for the square $(t=1)$ for $\gamma=60^{\circ}$. As discussed in [4] the zero Bond-number solution of a spherical cap is the asymptotic solution 
correct to $O(B)$ for the disk [6]. This holds also for the square domain, as proved in [4] and as indicated in Table 1.

In Table 1 solutions for $w$ obtained by straightforward use of PLTMG for meshes with three different numbers of vertices are tabulated at 11 equallyspaced points along a diagonal of the square domain $\Omega_{1}$ and along a radius of the disk domain $\Omega_{0}$. The asymptotic $(\mathrm{B}=0)$ solutions are tabulated $\left(w_{S}\right.$ for the square and $w_{D}$ for the disk), as well as the difference with the calculated $B=0.0001$ solution for each mesh size. One sees that the values for the square appear to be in accord with the asymptotic solution $w_{S}$, differing from it by more or less $O(B)$. Dependence of error on mesh size suggests that the discretization error is adequately small for the comparison to be made.

However, for the unit disk, the average values of the computed solutions are significantly distant from approximating the value of zero, the average height $\bar{w}$ of a solution to the continuous problem. The table indicates that the computed solutions differ from the asymptotic $w_{D}$ by an additive constant; the near-spherical shapes are evidently computed satisfactorily, but are displaced upward. This displacement effect was observed to be substantially less for $B=0.01$ and for larger values of $B$.

PLTMG provides the option of displaying the lowest frequency eigenvector of the Jacobian matrix at the computed solution, in this case the constant vector, in accordance with the continuous problem. As there are discretization and quadrature errors in PLTMG at a curved boundary that are not 
Table 1: Comparison of $B=0$ spherical solutions for $2 \times 2$ square $\left(w_{S}\right)$ and for unit disk $\left(w_{D}\right)$ with computed solutions $w$ for $B=0.0001$, for several mesh sizes.

\begin{tabular}{cc||cc|rr|rr}
\hline \hline \multicolumn{7}{c}{$2 \times 2$ Square } \\
\multicolumn{7}{c}{1995 vertices } \\
$r$ & $w_{S}$ & $w$ & $w-w_{S}$ & $w$ & $w-w_{S}$ & $w$ & $w-w_{S}$ \\
0.0000 & -0.1781 & -0.1785 & -0.0005 & -0.1785 & -0.0004 & -0.1782 & -0.0001 \\
0.1414 & -0.1731 & -0.1729 & 0.0001 & -0.1731 & 0.0000 & -0.1731 & 0.0000 \\
0.2828 & -0.1580 & -0.1580 & 0.0000 & -0.1579 & 0.0001 & -0.1579 & 0.0000 \\
0.4243 & -0.1326 & -0.1326 & 0.0000 & -0.1325 & 0.0001 & -0.1325 & 0.0000 \\
0.5657 & -0.0964 & -0.0963 & 0.0001 & -0.0964 & 0.0000 & -0.0964 & 0.0000 \\
0.7071 & -0.0489 & -0.0493 & -0.0004 & -0.0490 & -0.0001 & -0.0490 & -0.0001 \\
0.8485 & 0.0109 & 0.0111 & 0.0003 & 0.0108 & -0.0001 & 0.0108 & 0.0000 \\
0.9899 & 0.0841 & 0.0838 & -0.0003 & 0.0841 & 0.0000 & 0.0840 & -0.0001 \\
1.1314 & 0.1727 & 0.1724 & -0.0003 & 0.1727 & 0.0000 & 0.1726 & -0.0001 \\
1.2728 & 0.2792 & 0.2787 & -0.0005 & 0.2790 & -0.0002 & 0.2791 & -0.0001 \\
1.4142 & 0.4077 & 0.4062 & -0.0015 & 0.4065 & -0.0012 & 0.4073 & -0.0005 \\
& & & & & & & \\
\hline
\end{tabular}

Unit Disk

\begin{tabular}{cc||cc|cc|cc}
\multicolumn{1}{c}{2002 vertices } & \multicolumn{2}{c}{4000 vertices } & \multicolumn{2}{c}{5933 vertices } \\
$r$ & $w_{D}$ & $w$ & $w-w_{D}$ & $w$ & $w-w_{D}$ & $w$ & $w-w_{D}$ \\
0.0000 & -0.1308 & 2.2461 & 2.3769 & 0.8340 & 0.9648 & 0.4810 & 0.6118 \\
0.1000 & -0.1283 & 2.2489 & 2.3772 & 0.8366 & 0.9651 & 0.4836 & 0.6119 \\
0.2000 & -0.1207 & 2.2564 & 2.3772 & 0.8441 & 0.9651 & 0.4911 & 0.6119 \\
0.3000 & -0.1081 & 2.2689 & 2.3770 & 0.8567 & 0.9651 & 0.5037 & 0.6118 \\
0.4000 & -0.0904 & 2.2869 & 2.3772 & 0.8745 & 0.9651 & 0.5215 & 0.6119 \\
0.5000 & -0.0673 & 2.3098 & 2.3771 & 0.8976 & 0.9651 & 0.5446 & 0.6118 \\
0.6000 & -0.0386 & 2.3385 & 2.3771 & 0.9262 & 0.9651 & 0.5732 & 0.6118 \\
0.7000 & -0.0043 & 2.3729 & 2.3772 & 0.9607 & 0.9652 & 0.6076 & 0.6118 \\
0.8000 & 0.0362 & 2.4134 & 2.3772 & 1.0011 & 0.9651 & 0.6481 & 0.6119 \\
0.9000 & 0.0832 & 2.4605 & 2.3773 & 1.0481 & 0.9652 & 0.6951 & 0.6119 \\
1.0000 & 0.1372 & 2.5146 & 2.3774 & 1.1022 & 0.9652 & 0.7491 & 0.6119 \\
\hline \hline
\end{tabular}


present at a straight boundary, the evidence was that for the disk the poor conditioning of the matrix resulted in a considerable amount of constant vector contamination in the solutions. The discretization error approximating the curved boundary is greater the coarser the mesh, which is reflected in the corresponding contaminations shown for the unit disk in the table.

To correct for this behavior, we used the built-in features of PLTMG to subtract from the computed solution an appropriate multiple of the constant vector to yield an average height of zero, as required for a solution of the continuous problem (5),(6). This was done for all domains and for all Bond numbers, although the effect of the procedure was discernible just for the domain with curved boundaries and for the smallest Bond numbers. Once the corrected $w$ is calculated, the actual height $u$ is obtained from (4).

\section{$4.2 \quad$ Numerical Results}

The numerical results for the test problems are illustrated in Figs. 6 and 7. In Fig. 6 the liquid surface height difference between $\Omega_{1}$ and $\Omega_{t}$ at their center, denoted by $u_{1}(0)-u_{t}(0)$, is shown for each of the values of $t$ and $B$. The tabular values are connected with piecewise cubic splines. The ordinate is plotted on a logarithmic scale, to take account of the greatly changing behavior that occurs among the values of $B$, and to bring out the singular nature of the transition to the inscribed disk. The indicated increment upward by 0.1 allows the negative values of $u_{1}(0)-u_{t}(0)$ at $t=0$ to be displayed conveniently on the graph. 


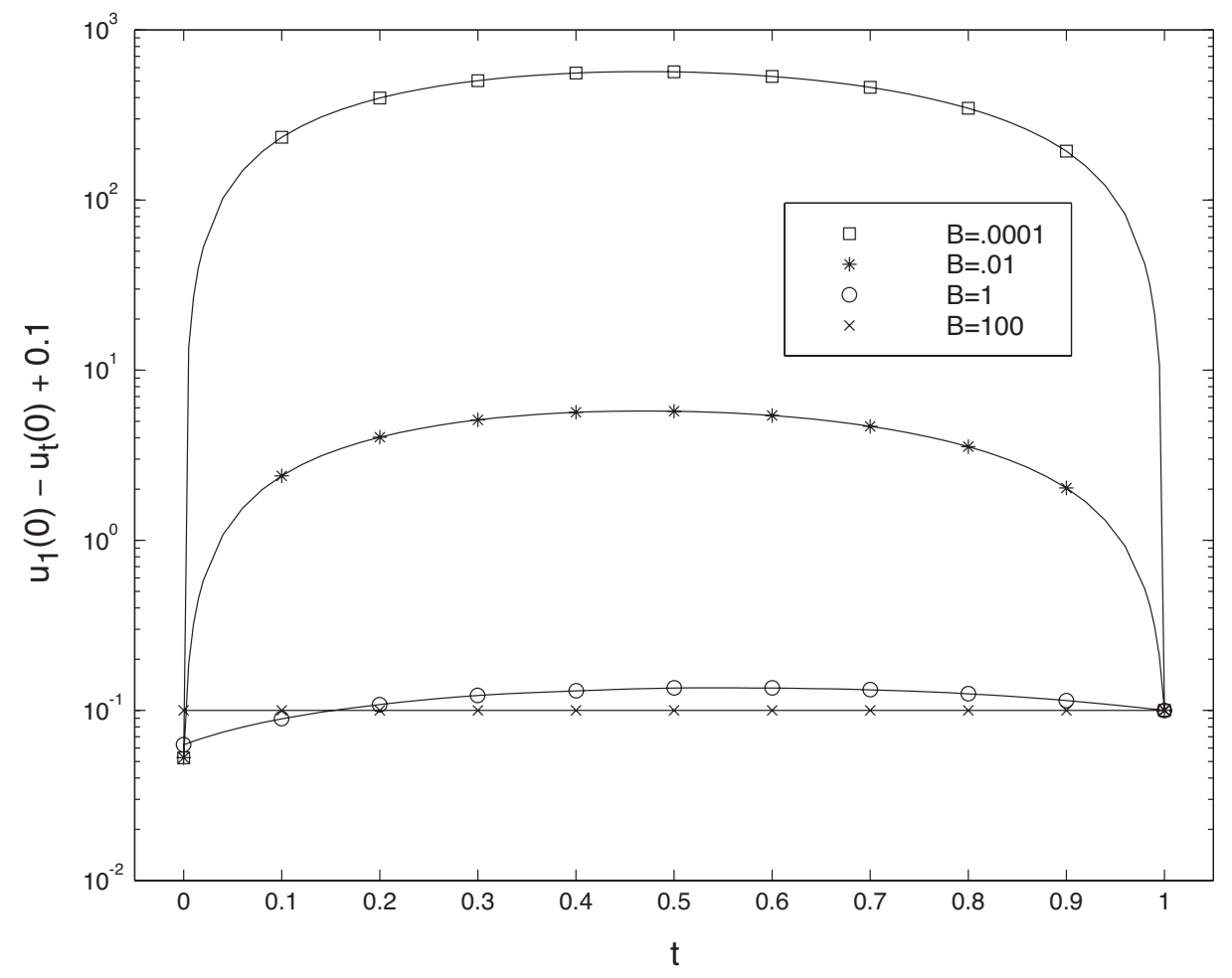

Figure 6: Surface height differences between $\Omega_{1}$ and $\Omega_{t}$ at their center vs. rounding parameter $t$ for several values of Bond number $B$.

In accordance with properties (i)-(iii) the square lifts liquid higher at its center than do the disq domains for small enough $B$, but does not lift liquid higher than the disk (note the negative values at $t=0$ ). As $B$ decreases, the height differences get very large. The large slopes at end points when $B$ is small suggests the discontinuous limiting behavior at $t=0$. A similar behavior in slope occurs near $t=1$, indicating there also the effect as $|\Sigma| /|\Omega|$ departs from the value 2 .

Fig. 7 depicts the height differences from the center to the boundary as a function of distance $s$ along a symmetry diagonal of $\Omega_{t}$, for the indicated 
Bond numbers. The first column gives the height difference between the square and the disq with rounding radius $1 / 2, u_{1}(s)-u_{\frac{1}{2}}(s)$. The positivity for small-enough $B$, in this case for the values other than $B=100$, and the very large values for the smallest B are in accord with property (i) of Sec. 2. The second column gives $u_{0}(s)-u_{\frac{1}{2}}(s)$. These values are all positive in accordance with property (ii), and again become very large for the smallest $B$. Note that the difference of ordinates between the first and second columns approach the asymptotic value for $B=0$ of the difference of the values of the constants in (7) of approximately $1.822-1.869=-0.047$. The third column of Fig. 7 gives the computed values of $u_{0}(s)-\hat{u}_{\frac{1}{2}}(s)$, where

$\hat{u}_{\frac{1}{2}}(s)$ is the solution of $(1),(2)$ in the domain $\hat{\Omega}_{\frac{1}{2}}$ obtained by shrinking $\Omega_{\frac{1}{2}}$ until its area equals that of $\Omega_{0}$. These curves should be compared directly with the corresponding ones for $u_{0}(s)-u_{\frac{1}{2}}(s)$ in the second column. As predicted (property (iv)) the height inequalities become very large in opposite directions as $B \rightarrow 0$.

\section{Acknowledgments}

We wish to thank R. E. Bank for advising us on the use of his PLTMG software package, in particular for suggesting how to take advantage of the built-in features of PLTMG to correct for contamination with the constant vector in the ill-conditioned case. Part of this work was supported by the National Science Foundation under Grants DMS-0103954 and DMS-0103937 and was carried out with computing facilities supported by the Office of Science of 

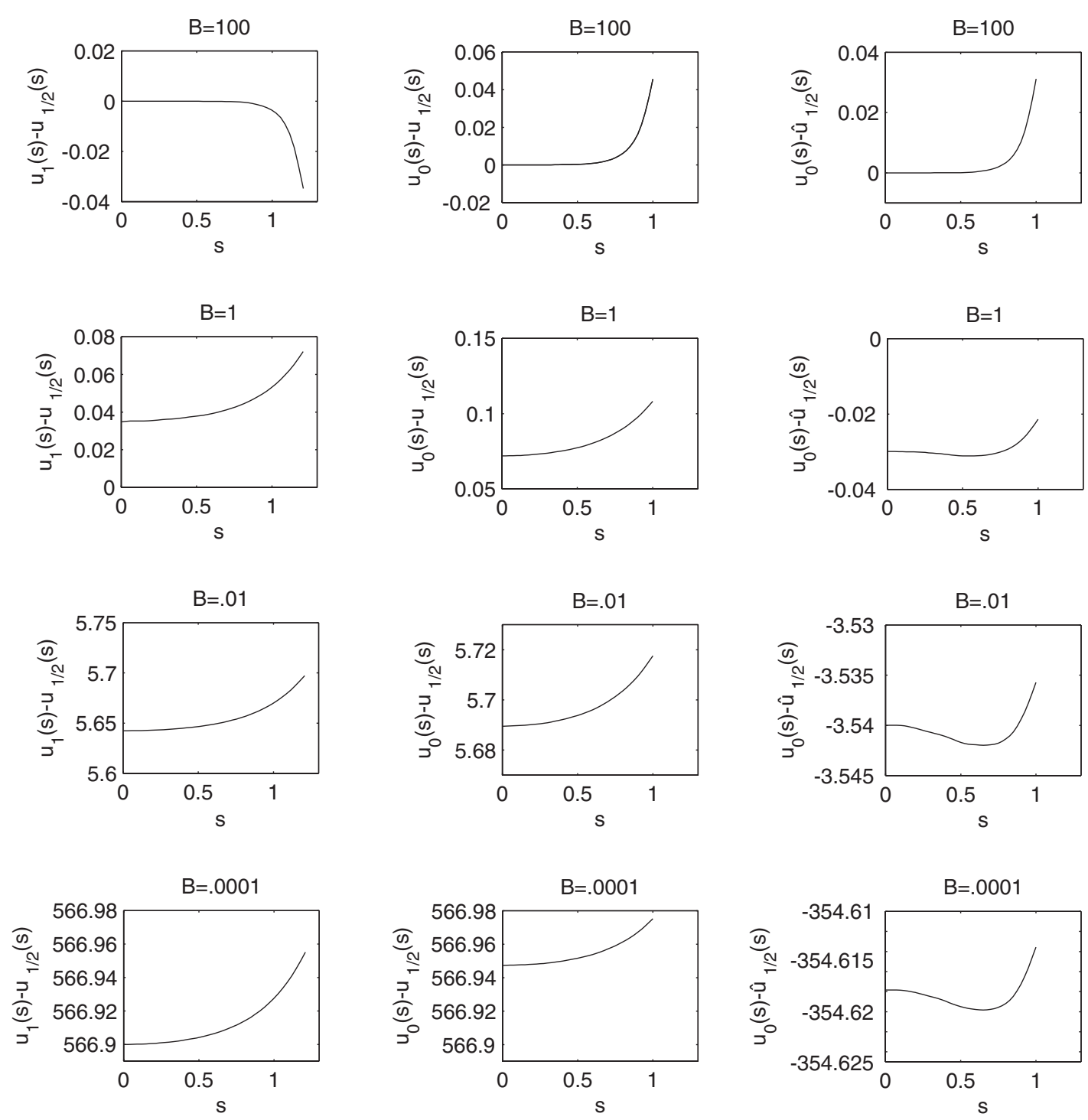

Figure 7: Surface height differences for: $\Omega_{1}$ and $\Omega_{1 / 2}$ (first column), $\Omega_{0}$ and $\Omega_{1 / 2}$ (second column), $\Omega_{0}$ and $\hat{\Omega}_{1 / 2}$ (third column). $B=100,1, .01, .0001$. 
the U.S. Department of Energy under Contract DE-AC03-76SF00098, which we gratefully acknowledge.

\section{References}

[1] R.E. Bank, PLTMG: A Software Package for Solving Elliptic Partial Differential Equations, Users' Guide 8.0, SIAM, 1988. Software available via Netlib at http://www.netlib.org/pltmg .

[2] P. Concus And R. Finn, On the height of a capillary surface, Math. Zeit., 147 (1976), pp. 93-95.

[3] R. Finn, Equilibrium Capillary Surfaces, Springer-Verlag, 1986; Russian translation: Mir Publishers, 1988.

[4] R. Finn and A.A. Kosmodem'yanskit, Jr., Some unusual comparison properties of capillary surfaces, Pacific J. Math., 205 (2002), pp. 119-137.

[5] J. JuRIN, An account of some experiments shown before the Royal Society; with an enquiry into the cause of the ascent and suspension of water in capillary tubes, Philos. Trans. R. Soc. London, 30 (1718), pp. $739-747$.

[6] D. Siegel, Height estimates for capillary surfaces, Pacific J. Math., 88 (1980), pp. 471-516.

[7] D. Siegel, The behavior of a capillary surface for small Bond number, in Variational Methods for Free Surface Interfaces, eds. P. Concus and R. Finn, Springer-Verlag, 1987, pp. 109-113. 